\title{
SUFFICIENT CONDITIONS FOR THE EXISTENCE OF CLASSICAL SOLUTION OF QUASI-INVERSE PROBLEM
}

\author{
Raid Almomani ${ }^{1}$, Ahmad Almomani ${ }^{2} \S$ \\ ${ }^{1}$ Department of Mathematics \\ Yarmouk University \\ Irbid - 21163, JORDAN \\ 21 College Circle, Mathematics Department \\ SUNY Geneseo, Geneseo, NY, 14454, USA
}

\begin{abstract}
In [1], the authors established a priori estimate for the solution of quasi-inverse problem for different weight functions, and they established a priori estimate for a higher order of the same problem. In this paper we get the generalized a priori estimation for the uniqueness solution of quasiinverse problems and we get the sufficient conditions for the existence of classical solution of quasi-inverse problems, such a problem play an important role in optimal control theory. Many works were devoted to this problem and the control problem of heat conduction with inverse direction of time and integral boundary conditions $[4,5,6,7,8,9]$.
\end{abstract}

AMS Subject Classification: $35 \mathrm{R} 30$

Key Words: nonlocal boundary conditions; quasi-inverse problem; a priori estimations

\section{Generalized a priori estimations. The uniqueness of solution of quasi-inverse problem}

We consider the quasi-inverse problem in the domain $Q=\{(x, t), 0<x<$ $1,0<t<T\}$

Received: December 13, 2019

(C) 2020 Academic Publications

${ }^{\S}$ Correspondence author 


$$
\begin{gathered}
\frac{\partial U_{\epsilon}}{\partial t}+\frac{\partial^{2} U_{\epsilon}}{\partial x^{2}}-\epsilon \frac{\partial^{3} U_{\epsilon}}{\partial x^{2} \partial t}=0, \quad(x, t) \in Q, t>0 \\
U_{\epsilon}(x, 0)=X(x), \quad 0<x<1 \\
U_{\epsilon}(0, t)=0, \int_{0}^{1} U_{\epsilon}(x, t) d x=0 .
\end{gathered}
$$

We assume that $X(x) \in W_{2}^{1}(0,1)$ satisfies the conditions

$$
X(0)=0, \int_{0}^{1} X(x) d x=0 .
$$

We will unite the a priori estimations established in $[2,3]$ by a single representation. To do so, we introduce the solution space $E^{K, L}$ and the space of the right-hand side $F^{K, L}$ of problem (1) with the following norms

$$
\begin{gathered}
\left\|\mid U_{\epsilon}\right\|_{K, L}^{2}=\int_{Q}(1-x)^{k}\left(\frac{\partial^{L+1} U_{\epsilon}}{\partial x^{L} \partial t}\right)^{2} d x d t \\
+\frac{\epsilon}{2} \int_{Q}(1-x)^{k}\left(\frac{\partial^{L+2} U_{\epsilon}}{\partial x^{L+1} \partial t}\right)^{2} d x d t, \\
\|x\|_{K, L}^{2}=\int_{0}^{1}(1-x)^{k}\left(\frac{\partial^{L+1} x}{\partial x^{L+1}}\right)^{2} d x,
\end{gathered}
$$

where $K=1,2 ; L=0,1 ; K>L$. Thus, the results of $[2,3]$ can be formulated in the following theorem.

Theorem 1. For the solution of (1) the following a priori estimations holds

$$
\left\|\left.\left|U_{\epsilon}\right|\right|_{K, L} ^{2} \leq C_{K, L}\right\| x \|_{K, L}^{2}
$$

where

$$
C_{K, L}=\left(2^{K+L-1}+K+L-1\right)^{2} \frac{T}{\epsilon} e^{2\left(2^{K+L-1}+K+L-1\right)^{2} \frac{T^{2}}{e^{2}}}
$$

$K=1,2, L=0,1, K>L$.

Conclusion 1. If the solution of problem (1) exists, then it is unique.

For proof, it is sufficient to verify that the solution of the homogeneous problem of (1) is trivial, which implies from (5).

Conclusion 2. The solution of (1) continuously depends on the functions $X(x)$. 
From (5) it follows that a small change in the initial functions $X(x)$ in the norms of the space $F^{K, L}$ corresponds to a small change of the solution $U_{\epsilon}(x, t)$ Of (1) in the norms of the space $E^{K, L}$. It is well known that the sequence of functions

$$
X_{0}(x)=x, X_{2 k-1}(x)=x \cdot \cos (2 \pi k x), X_{2 k}=\sin (2 \pi k x), k=1,2, \ldots,
$$

are the eigenfunctions and adjoint functions of the corresponding Sturm-Liouville problem and they form a basis in the space $L_{2}(0,1)$. We will write the expansion of $X(x)$ in biorthogonal series by

$$
X(x)=\varphi_{0} X_{0}(x)+\sum_{k=1}^{\infty} \varphi_{2 k} X_{2 k}(x)+\varphi_{2 k-1} X_{2 k-1}(x),
$$

where

$$
\begin{gathered}
\varphi_{0}=\int_{0}^{1} X(x) Y_{0}(x) d x, \\
\varphi_{2 k}=\int_{0}^{1} X(x) Y_{2 k}(x) d x, \\
\varphi_{2 k-1}=\int_{0}^{1} X(x) Y_{2 k-1}(x) d x, \\
Y_{0}(x)=2, Y_{2 k-1}(x)=4 \cdot \cos (2 \pi k x), \\
Y_{2 k}(x)=4(1-x) \sin (2 \pi k x), k=1,2, \ldots,
\end{gathered}
$$

are the eigenfunctions and adjoint functions of the conjugate Sturm-Liouville problem. In particular, and since $\int_{0}^{1} X(x) d x=0$, then in $(8) \varphi_{0}=0$.

By analogy with the Fourier method, we will seek the solution of (1) in the form

$$
U_{\epsilon}(x, t)=\sum_{k=1}^{\infty} \varphi_{2 k}(t) X_{2 k}(x)+\varphi_{2 k-1}(t) X_{2 k-1}(x),
$$

where $\varphi_{2 k}(t)$ and $\varphi_{2 k-1}(t)$ are unknown functions.

Substituting (11) into (1), assuming the possibility of term by term differentiation of (11) with respect to the variables $x$ and $t$, and taking into account that for the eigenfunctions and adjoint functions the following hold

$$
\begin{gathered}
X_{2 k}^{\prime \prime}=-\lambda_{k} X_{2 k}(x) \\
X_{2 k-1}^{\prime \prime}=-\lambda_{k} X_{2 k-1}(x)-2 \sqrt{\lambda_{k}} X_{2 k}(x),
\end{gathered}
$$


where $\lambda_{k}=(2 \pi k)^{2}, k=1,2, \ldots$ are the eigenvalues of Sturm-Liouville problem, using the initial condition of (1), we arrive to the following system of ODE's with unknown functions $\varphi_{2 k}(t)$ and $\varphi_{2 k-1}(t)$,

$$
\begin{gathered}
\varphi_{2 k}^{\prime}(t)\left(1+\lambda_{k} \epsilon\right)-\lambda_{k} \varphi_{2 k}(t)-2 \sqrt{\lambda_{k}}\left(\varphi_{2 k-1}(t)-\epsilon \varphi_{2 k}^{\prime}\right)=0, \\
\varphi_{2 k-1}^{\prime}(t)\left(1+\lambda_{k} \epsilon\right)-\lambda_{k} \varphi_{2 k-1}(t)=0, \\
\varphi_{2 k}(0)=\varphi_{2 k}, \varphi_{2 k-1}(0)=\varphi_{2 k-1},
\end{gathered}
$$

which has the following solution

$$
\begin{gathered}
\varphi_{2 k-1}(t)=\varphi_{2 k-1} e^{\frac{\lambda_{k}}{1+\lambda_{k} \epsilon} t}, \\
\varphi_{2 k}(t)=\left(\frac{2 \sqrt{\lambda_{k}}}{\left(1+\lambda_{k} \epsilon\right)^{2}} \varphi_{2 k-1} t+\varphi_{2 k}\right) e^{\frac{\lambda_{k}}{1+\lambda_{k}} \epsilon} .
\end{gathered}
$$

Thus, the unknown solution $U_{\epsilon}(x, t)$ of problem (1) has the following form

$$
\begin{aligned}
U_{\epsilon}(x, t)=\sum_{k=1}^{\infty}\left[\left(\frac{2 \sqrt{\lambda_{k}}}{\left(1+\lambda_{k} \epsilon\right)^{2}} \varphi_{2 k-1} t+\varphi_{2 k}\right)\right. & X_{2 k}(x) \\
& \left.+\varphi_{2 k-1} X_{2 k-1}(x)\right] e^{\frac{\lambda_{k}}{1+\lambda_{k} \epsilon} t},
\end{aligned}
$$

where $X_{2 k}(x)=\sin (2 \pi k x), X_{2 k-1}(x)=x \cdot \cos (2 \pi k x), \lambda_{k}=(2 \pi k)^{2}$.

\section{Sufficient conditions for the existence of classical solution of quasi-inverse problem}

Theorem 2. Let the function $X(x) \in W_{2}^{3}(0,1)$ satisfy the following conditions

$$
\begin{aligned}
& X(0)=0, \int_{0}^{1} X(x) d x=0, \\
& X^{\prime}(0)=X^{\prime}(1), X^{\prime \prime}(0)=0 .
\end{aligned}
$$

Then the function $U_{\epsilon}(x, t)$ defined by the biorthogonal series (14), where the coefficients $\varphi_{2 k}, \varphi_{2 k-1}$ are defined by (9) is the classical solution of problem (1).

We get a formal solution $U_{\epsilon}(x, t)$ of problem (1) written in the form of biorthogonal series (11). To make sense of the initial and boundary condition of problem (1), the function $U_{\epsilon}(x, t)$ must be continuous in the closed domain

$$
\bar{Q}=\{(x, t), 0 \leq x \leq 1,0 \leq t \leq T\} .
$$


Thus, it is sufficient for the solution of (1), that the series (11) and the following series

$$
\sum_{k=1}^{\infty} \frac{\partial U_{\epsilon, k}}{\partial t}, \sum_{k=1}^{\infty} \frac{\partial^{2} U_{\epsilon, k}}{\partial x^{2}}, \sum_{k=1}^{\infty} \frac{\partial^{3} U_{\epsilon, k}}{\partial x^{2} \partial t},
$$

(where $U_{\epsilon, k}(x, t)$ is the $k$-th term of the series (14)), were uniformally and absolutely convergent in $\bar{Q}$. For this, it is sufficient to establish the uniformly and absolutely convergence of the series

$$
\sum_{k=1}^{\infty} \frac{\partial^{3} U_{\epsilon, k}}{\partial x^{2} \partial t}
$$

in $\bar{Q}$ for $\epsilon>0$. Note that, to solve the question of uniformly and absolutely convergence of (16) we need a redundant requirement which is the closure of the domain $\bar{Q}$, thus for such convergence it is sufficient to establish it for the domain $Q$,

$$
\begin{aligned}
& \frac{\partial^{3} U_{\epsilon, k}}{\partial x^{2} \partial t}=-\left\{\left[\left(\frac{2 \sqrt{\lambda_{k}} \lambda_{k}}{\left(1+\lambda_{k} \epsilon\right)^{2}} \varphi_{2 k-1} t+\lambda_{k} \varphi_{2 k}+2 \sqrt{\lambda_{k}} \varphi_{2 k-1}\right) X_{2 k}(x)\right.\right. \\
& \left.\left.+\lambda_{k} \varphi_{2 k-1} X_{2 k-1}(x)\right] \frac{\lambda_{k}}{1+\lambda_{k} \epsilon}+\frac{2 \sqrt{\lambda_{k}} \lambda_{k}}{\left(1+\lambda_{k} \epsilon\right)^{2}} \varphi_{2 k-1} X_{2 k}(x)\right\} e^{\frac{\lambda_{k}}{1+\lambda_{k} \epsilon} t} .
\end{aligned}
$$

Then for $\forall(x, t) \in \bar{Q}, \epsilon>0$, we have the following estimation

$$
\begin{aligned}
\left|\frac{\partial^{3} U_{\epsilon, k}}{\partial x^{2} \partial t}\right| \leq\{ & \left\{\left(\frac{2}{\epsilon^{2}} \frac{1}{\sqrt{\lambda_{k}}} T\left|\varphi_{2 k-1}\right|+\lambda_{k}\left|\varphi_{2 k}\right|+2 \sqrt{\lambda_{k}}\left|\varphi_{2 k-1}+\lambda_{k}\right| \varphi_{2 k-1} \mid\right) \frac{1}{\epsilon}\right. \\
& \left.+\frac{2}{\epsilon^{2}} \frac{1}{\sqrt{\lambda_{k}}}\left|\varphi_{2 k-1}\right|\right\} e^{\frac{T}{\epsilon}} \leq M k^{2}\left(\left|\varphi_{2 k}\right|+\left|\varphi_{2 k-1}\right|\right),
\end{aligned}
$$

where the positive constant $M$ does not depend on $k$. We estimate $\left|\varphi_{2 k}\right|$ and $\left|\varphi_{2 k-1}\right|$. A formula for $\varphi_{2 k-1}$ from (9), we integrate twice by parts

$$
\begin{aligned}
& \varphi_{2 k-1}=\int_{0}^{1} X(x) Y_{2 k-1}(x) d x=4 \int_{0}^{1} X(x) \cos (2 \pi k x) d x \\
& =\frac{1}{\pi^{2} k^{2}}\left(\left.X^{\prime}(x) \cos (2 \pi k x)\right|_{0} ^{1}-\int_{0}^{1} X^{\prime \prime}(x) \cos (2 \pi k x) d x\right),
\end{aligned}
$$

we require that

$$
X^{\prime}(0)=X^{\prime}(1),
$$


and once again we integrate by parts, we get

$$
\varphi_{2 k-1}=\frac{1}{2 \pi^{3} k^{3}} \int_{0}^{1} X^{\prime \prime \prime}(x) \sin (2 \pi k x) d x,
$$

we set $\left|\varphi_{2 k-1}\right|$ in the following form

$$
\left|\varphi_{2 k-1}\right|=\frac{1}{k^{2}} \frac{1}{2 \sqrt{2} \pi^{3}} \frac{\left|a_{k}\right|}{k},
$$

where

$$
a_{k}=\sqrt{2} \int_{0}^{1} X^{\prime \prime \prime}(x) \sin (2 \pi k x) d x .
$$

By analogy with a formula for $\varphi_{2 k}$ from (9), we integrate three times by parts taking into account that $X(0)=0, X^{\prime}(0)=X^{\prime}(1)$, and requiring that

$$
X^{\prime \prime}(0)=0
$$

we get

$$
\begin{gathered}
\varphi_{2 k}=\int_{0}^{1} X(x) Y_{2 k}(x) d x=4 \int_{0}^{1} X(x)(1-x) \sin (2 \pi k x) d x \\
=\frac{-1}{2 \pi^{3} k^{3}} \int_{0}^{1}\left[X^{\prime \prime \prime}(x)(1-x)-3 x^{\prime \prime}(x)\right] \cos (2 \pi k x) d x \\
=\frac{-1}{2 \sqrt{2} \pi^{3} k^{3}} \int_{0}^{1}(1-x) X^{\prime \prime \prime}(x) \sqrt{2} \cos (2 \pi k x) d x \\
\quad+\frac{3}{2 \sqrt{2} \pi^{3} k^{3}} \int_{0}^{1} X^{\prime \prime}(x) \sqrt{2} \cos (2 \pi k x) d x
\end{gathered}
$$

Thus,

$$
\left|\varphi_{2 k}\right| \leq \frac{1}{k^{2}} \frac{1}{2 \sqrt{2} \pi^{3}}\left(\frac{b_{k}}{k}+\frac{3\left(c_{k}\right)}{k}\right)
$$

where

$$
\begin{gathered}
b_{k}=\int_{0}^{1}(1-x) X^{\prime \prime \prime}(x) \sqrt{2} \cos (2 \pi k x) d x, \\
c_{k}=\int_{0}^{1} X^{\prime \prime}(x) \sqrt{2} \cos (2 \pi k x) d x .
\end{gathered}
$$


To the right hand parts of (19) and (22) we apply inequality about geometric and arithmetic means, we estimate from above inequality (17),

$$
k^{2}\left(\left|\varphi_{2 k}\right|+\left|\varphi_{2 k-1}\right|\right) \leq \frac{1}{4 \sqrt{2} \pi^{3}}\left(a_{k}^{2}+b_{k}^{2}+c_{k}^{2}+\frac{11}{k^{2}}\right) .
$$

Since $a_{k}, b_{k}$, and $c_{k}$ are the Fourier coefficients of the functions $X^{\prime \prime \prime}(x),(1-$ $x) X^{\prime \prime \prime}(x)$, and $X^{\prime \prime}(x)$ respectively by orthonormalized trigonometric functions system on $[0,1]$

$$
\sqrt{2} \cdot \cos (2 \pi k x), \sqrt{2} \cdot \sin (2 \pi k x), k=1,2, \ldots,
$$

then for these functions the following inequalities hold

$$
\begin{gathered}
\sum_{k=1}^{\infty} a_{k}^{2} \leq\left\|X^{\prime \prime \prime}\right\|_{L_{2}(0,1)}^{2}=\int_{0}^{1}\left(X^{\prime \prime \prime}(x)\right)^{2} d x \\
\sum_{k=1}^{\infty} b_{k}^{2} \leq\left\|(1-x) X^{\prime \prime \prime}(x)\right\|_{L_{2}(0,1)^{2}}=\int_{0}^{1}\left(X^{\prime \prime \prime}(x)\right)^{2} d x \\
\leq \int_{0}^{1}\left(X^{\prime \prime \prime}(x)\right)^{2} d x \\
\sum_{k=1}^{\infty} c_{k}^{2} \leq\left\|X^{\prime \prime}(x)\right\|_{L_{2}(0,1)^{2}}=\int_{0}^{1}\left(X^{\prime \prime}(x)\right)^{2} d x
\end{gathered}
$$

we require that

$$
X^{\prime \prime}(x) \in L_{2}(0,1), X^{\prime \prime \prime}(x) \in L_{2}(0,1) .
$$

Then from inequality (26) implies the convergence of the series

$$
\sum_{k=1}^{\infty} a_{k}^{2}, \sum_{k=1}^{\infty} b_{k}^{2}, \sum_{k=1}^{\infty} c_{k}^{2}
$$

from inequality (22) implies the convergence of the series

$$
\sum_{k=1}^{\infty} k^{2}\left(\left|\varphi_{2 k}\right|+\left|\varphi_{2 k-1}\right|\right)
$$

and from inequality (17) by the Weierstrass test implies the uniformly and absolutely convergence of (16),

$$
\sum_{k=1}^{\infty} \frac{\partial^{3} U_{\epsilon, k}}{\partial x^{2} \partial t}
$$


which means, also the series (15),

$$
\sum_{k=1}^{\infty} \frac{\partial^{2} U_{\epsilon, k}}{\partial x^{2}}, \sum_{k=1}^{\infty} \frac{\partial U_{\epsilon, k}}{\partial t}, \sum_{k=1}^{\infty} U_{\epsilon, k}
$$

in the domain $\bar{Q}, \forall \epsilon>0$. Thus, by acceptable restriction on $X(x)$, the function $U_{\epsilon}(x, t)$ defined by the biorthogonal series (14) is

- Continuous in the domain $\bar{Q}$.

- Have in $\bar{Q}$ continuous partial derivatives $\frac{\partial U_{\epsilon}}{\partial t}, \frac{\partial^{2} U_{\epsilon}}{\partial x^{2}}, \frac{\partial^{3} U_{\epsilon}}{\partial x^{2} \partial t}$, that can be found by term by term differentiation of the series (14).

- Satisfy the differential equation, initial conditions, and boundary condition of problem (1).

Consequently, this function $U_{\epsilon}(x, t)$ is the classical solution of problem (1) in the domain $\bar{Q}(\epsilon>0)$.

\section{References}

[1] R. Almomani, H. Almefleh, On heat conduction problem with integral boundary condition, J. of Emerging Trends in Eng. and Appl. Sci. (JETEAS), 3, No 6 (2012), 977-979.

[2] H. Almefleh, R. Almomani, New a priori estimations of the solution of quasi-inverse problem, International Journal of Applied Mathematics, 27, No 3 (2014), 205-210; DOI: 10.12732/ijam.v27i3.1.

[3] A. Almomani, R. Almomani, Higher order estimation of the solution of quasi-inverse problem, International Journal of Applied Mathematics, 32, No 4 (2019), 623-626; DOI: 10.12732/ijam.v32i4.6.

[4] N. E. Benouar and N. I. Yurchuk, Mixed problem with an integral condition for parabolic equations with the Bessel operator, Dierentsial'nye Uravneniya, 27 (1991), 2094-2098.

[5] A. Bouziani, Mixed problem with boundary integral conditions for a certain parabolic equation, J. Appl. Math. Stochastic Anal., 9 (1996), 323-330.

[6] A. Bouziani, Strong solution for a mixed problem with nonlocal condition for a certain pluriparabolic equations, Hiroshima Math. J., 27 (1997), 373390. 
[7] M. Feng, B. Du, W. Ge, Impulsive boundary value problems with integral boundary conditions and one-dimensional p-Laplacian, Nonlinear Anal., 70 (2009), 3119-3126.

[8] N.I. Ionkin, Solution of a boundary-value problem in heat conduction with a nonclassical boundary condition, Diff. Eqs., 13, No 2 (1977), 294-304.

[9] N.I. Yurchuk, Mixed problem with an integral condition for certain parabolic equations, Diff. Eqs., 22, No 12 (1986), 2117-2126. 
